\title{
Effectiveness of different instant hand sanitizers against normal flora and some selected pathogenic bacteria
}

\author{
Tasnia Ahmed* \\ Department of Microbiology, Stamford University Bangladesh, 51, Siddeswari Road, Dhaka 1217, Bangladesh
}

Received 04 March 2018/Accepted 29 March 2018

\begin{abstract}
To maintain hygienic life it is important to follow the rules of sanitation. Hand sanitation is one of the most necessary parts of keeping personal hygiene. As hands are used directly for person to person contact, contact with animals, food preparation and so on, they can transmit microorganisms to and from all of these sources coming in contact. So if proper hand sanitation is maintained, the transfer of microorganisms will be decreased which can ultimately reduce the transmission of some pathogenic microorganisms to a susceptible host from own self directly or from the animals after handling them. Susceptible people getting harmful pathogenic bacteria can get sick if they find their appropriate routes of entry into them. During food preparation and taking meals, microorganisms can get entrance into the body from hands as well as microbes contaminating foods from the hands can proliferate in the foods causing spoilage and also release toxins causing food borne infection and food borne intoxication respectively. To reduce such risks people need to wash hands on routine basis especially on food processing zone it should be washed with water and soap which results in greater cleaning. People now use instant hand sanitizer after washing hands with water giving an extra protection. Sanitizers works best on hands with no visible sign of dirt. In the current study, five alcohol based hand sanitizers were selected to determine the ability of these sanitizers to decrease the loads of microorganisms from the hands of the five selected volunteers. During the study, it has been found that alcohol based instant hand sanitizer do decrease the loads of microbial flora from the hands but not in satisfactory level. Even they possessed very low effectivity against some pathogenic bacteria. These products can be used only where water is not available to decrease the loads of microbes from the hands.
\end{abstract}

Key words: Hand sanitizer; Personal hygiene; Normal flora; Pathogenic bacteria

In maintaining good hygienic life it is important to know the ways of hygiene. The term is often related to the sanitation and cleanliness starting from one's body and disseminating it to other day to day life activities as well. Making the body parts clean and germ free (pathogenic or spoilage causing bacteria) especially those parts which come into direct contact with other people as well as things used in life is the first way of the sanitation or good hygienic life (1). As we use hands directly for preparing and handling food as well as taking care of patients (immune compromised people), hands should be kept free from pathogenic and spoilage causing bacteria. The best way for this is to wash hands with soap and water and rubbing the hands during washing which wash off the unwanted germs from hands (2-6). Another popular way for hand sanitation is using instant hand sanitizers which are applied directly on hands where washing hands with water is not possible. Washing of hands is really important in maintaining personal hygiene and this should be strictly maintained in food preparation sectors and hospital settings. If not cleaned enough, hands may contribute to add pathogenic microorganisms in food items in kitchen

*Corresponding Author: Mailing address. Tasnia Ahmed, Senior Lecturer Department of Microbiology, Stamford University Bangladesh, 51 Siddeswari Road, Dhaka 1217, Bangladesh, Bangladesh; E-mail: tasnia2009@yahoo.com. and commercial food processing areas which can ultimately cause serious foodborne disease as well as foodborne intoxication (6-8).

Pathogenic bacteria can get into the mouth directly by hands if personal hygiene is not maintained. In hospitals, nurse and doctors have to use hand gloves during patient handling but sometimes not following the rules can contribute to the transmission microorganisms from hands to the patient. So proper hand washing can solve all of these problems. As instant hand sanitizer using is increasing in these days, there are still confusion about the effectiveness of it over conventional hand washing with water. During conventional hand washing people often use soaps without any antimicrobials or disinfectants.

Simply rubbing or friction and using water thoroughly can reduce the load of bacteria as well as dirt. But nowadays different antimicrobial agents (triclosan, parachloro-meta-xylinol, chlorhexidine gluconate) are used in soaps to improve the effectiveness of soap during hand washing which adds an extra advantage of killing or inhibiting bacteria but with lower effectivity for gram negative bacteria $(9,10)$. On the other hand, instant hand sanitizers can be both alcohol based or alcohol free products. Alcohol based sanitizers are made of about varying concentrations of alcohol (60\% to $95 \%)$ which 
are combined with thickening agents (propylene glycol, glycerin) to slower the rapid drying effect of alcohol after application on hands. These sanitizers have activity against bacteria, virus, fungi (11-13). Alcohol free hand sanitizers contain some disinfecting components like iodine, quaternary ammonium compounds etc. These are less effective than alcohol based sanitizers but the problem overcame with the use of benzalkonium chloride which has made alcohol free sanitizers as effective as alcohol based sanitizers (14). The effectivity of instant sanitizers depends on the concentration of alcohol/disinfectants, time of exposure, types and loads of microorganisms, presence of dirt or grease on hands etc. $(11,12,15-20)$. Hand washing with soap and water is more appreciated in food handling areas because hand sanitizer cannot work well if the targeted surface contains lots of dirt or greasy materials. As hands are directly used in different stages of food preparation and the consumers of different ages eat these foods, no compromise is accepted with their health (21-24). Hand washing is only preferable in such conditions (25). In case of hospitals, the health care personnel are not involved in direct food handling for the patients. They only take care of the patients externally. In this case, they can use hand sanitizers during investigating the patients. As in hospital settings, hands are not as dirty as food processing area because they used to wash hands in routine basis (low number of contaminants present on hands), hand sanitizers can act more effectively. This current study was aimed to determine by how much the loads of microorganisms (normal microbial flora) are decreased while using instant hand sanitizers without any prior conventional hand washing process. At the same time their ability to inhibit some pathogenic bacteria was also included in this study.

\section{MATERIALS AND METHOD}

Determining the efficacy of instant sanitizer in terms of decreasing the microbial load present on the hands without any prior hand washing with soap was the main concern of the current study. Five volunteers were selected randomly to conduct the experiment. Five different hand sanitizer samples were used to demonstrate their activity in decreasing microorganisms from the hands of the volunteers.

Sample before using instant hand sanitizer. At first, hands were rinsed with sterile distilled water. The water was used to inoculate onto different culture media like Nutrient Agar (NA), Sabouraud Dextrose Agar (SDA), Centrimide Agar (CA), Mannitol Salt Agar (MSA) followed by spreading with a spreader to determine the types of bacteria as well as to count their load per $\mathrm{ml}$ of rinsed water. The plates were incubated at $37{ }^{\circ} \mathrm{C}$ for 24 hours except SDA which were incubated at $25{ }^{\circ} \mathrm{C}$ for 48 hours.

Sample after using instant hand sanitizer. After the first wash and the firs round of inoculation, same volunteers were called up again to use instant hand sanitizer. The time interval was approximately thirty minutes between first hand wash with sterile water and the second round of hand sanitation. After application, the volunteers waited for ten minutes to let the sanitizer work on the microorganisms present on the application area and again washed with sterile distilled water. This water was used to inoculate onto different culture media (Nutrient Agar (NA), Sabouraud Dextrose Agar (SDA), Centrimide Agar (CA), Mannitol Salt Agar (MSA) etc.) like previous stage. After incubation plates were observed for the presence of the specific microorganism on specific media as well as compared the results of both hand rinsing water like before and after using hand sanitizer.

Determination of the inhibitory effect against known pathogenic bacteria. Some suspension of laboratory isolates were used to prepare a lawn over Mueller Hinton Agar (MHA) medium using sterile cotton swab. Then using a cork borer holes were made on the MHA to introduce hand sanitizer samples directly in it.

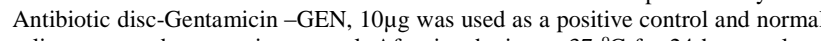
saline was used as negative control. After incubation at $37{ }^{\circ} \mathrm{C}$ for 24 hours, plates were observed for the presence of clear zone $(26,27)$.

\section{RESULTS AND DISCUSSION}

Five different alcohol based instant hand sanitizers were

TABLE 1. Microbial load in hand rinsed water of before and after using instant sanitizer sample 1

\begin{tabular}{|c|c|c|c|c|c|c|c|c|}
\hline \multirow[b]{2}{*}{ Volunteer } & \multicolumn{2}{|c|}{ Total bacterial count $(\mathrm{cfu} / \mathrm{ml})$} & \multicolumn{2}{|c|}{ Total fungal count (cfu/ml) } & \multicolumn{2}{|c|}{ Staphylococcus spp. (cfu/ml) } & \multicolumn{2}{|c|}{ Pseudomonas spp. $(\mathrm{cfu} / \mathrm{ml})$} \\
\hline & $\begin{array}{c}\text { Hand rinsed } \\
\text { water } \\
\text { before using } \\
\text { sanitizer }\end{array}$ & $\begin{array}{c}\text { Hand rinsed } \\
\text { water after } \\
\text { using } \\
\text { sanitizer }\end{array}$ & $\begin{array}{c}\text { Hand rinsed } \\
\text { water } \\
\text { before using } \\
\text { sanitizer }\end{array}$ & $\begin{array}{c}\text { Hand rinsed } \\
\text { water after } \\
\text { using } \\
\text { sanitizer }\end{array}$ & $\begin{array}{c}\text { Hand rinsed } \\
\text { water } \\
\text { before using } \\
\text { sanitizer }\end{array}$ & $\begin{array}{c}\text { Hand rinsed } \\
\text { water after } \\
\text { using } \\
\text { sanitizer }\end{array}$ & $\begin{array}{c}\text { Hand rinsed } \\
\text { water before } \\
\text { using } \\
\text { sanitizer }\end{array}$ & $\begin{array}{c}\text { Hand rinsed } \\
\text { water after } \\
\text { using } \\
\text { sanitizer }\end{array}$ \\
\hline 01 & $2.9 \times 10^{4}$ & $1.7 \times 10^{3}$ & $1.8 \times 10^{3}$ & $1.0 \times 10^{3}$ & $1.8 \times 10^{3}$ & $2.0 \times 10^{2}$ & $2.0 \times 10^{1}$ & - \\
\hline 02 & $2.1 \times 10^{4}$ & $1.5 \times 10^{3}$ & $1.0 \times 10^{3}$ & $1.5 \times 10^{2}$ & $1.8 \times 10^{3}$ & $1.1 \times 10^{3}$ & $8.0 \times 10^{2}$ & $5.0 \times 10^{1}$ \\
\hline 03 & $1.8 \times 10^{3}$ & $5.0 \times 10^{2}$ & $2.8 \times 10^{3}$ & $4.5 \times 10^{2}$ & $1.9 \times 10^{3}$ & $4.0 \times 10^{2}$ & - & - \\
\hline 04 & $2.2 \times 10^{4}$ & $7.5 \times 10^{2}$ & $2.7 \times 10^{3}$ & $5.2 \times 10^{2}$ & $5.0 \times 10^{3}$ & $1.0 \times 10^{2}$ & $5.0 \times 10^{1}$ & - \\
\hline 05 & $2.3 \times 10^{3}$ & $1.2 \times 10^{2}$ & $1.3 \times 10^{3}$ & $1.0 \times 10^{3}$ & $3.4 \times 10^{2}$ & $2.1 \times 10^{2}$ & $1.7 \times 10^{1}$ & - \\
\hline
\end{tabular}

TABLE 2. Microbial load in hand rinsed water of before and after using instant sanitizer sample 2

\begin{tabular}{|c|c|c|c|c|c|c|c|c|}
\hline \multirow[b]{2}{*}{ Volunteer } & \multicolumn{2}{|c|}{ Total bacterial count } & \multicolumn{2}{|c|}{ Total fungal count } & \multicolumn{2}{|c|}{ Staphylococcus spp. } & \multicolumn{2}{|c|}{ Pseudomonas spp. } \\
\hline & $\begin{array}{l}\text { Hand rinsed } \\
\text { water } \\
\text { before using } \\
\text { sanitizer }\end{array}$ & $\begin{array}{c}\text { Hand rinsed } \\
\text { water after } \\
\text { using sanitizer }\end{array}$ & $\begin{array}{l}\text { Hand rinsed } \\
\text { water } \\
\text { before using } \\
\text { sanitizer }\end{array}$ & $\begin{array}{c}\text { Hand rinsed } \\
\text { water after } \\
\text { using } \\
\text { sanitizer }\end{array}$ & $\begin{array}{l}\text { Hand rinsed } \\
\text { water } \\
\text { before using } \\
\text { sanitizer }\end{array}$ & $\begin{array}{l}\text { Hand rinsed } \\
\text { water after } \\
\text { using } \\
\text { sanitizer }\end{array}$ & $\begin{array}{l}\text { Hand rinsed } \\
\text { water before } \\
\text { using } \\
\text { sanitizer }\end{array}$ & $\begin{array}{c}\text { Hand rinsed } \\
\text { water after } \\
\text { using } \\
\text { sanitizer }\end{array}$ \\
\hline 01 & $1.9 \times 10^{3}$ & $5.0 \times 10^{2}$ & $1.5 \times 10^{3}$ & $1.0 \times 10^{2}$ & $1.8 \times 10^{2}$ & $1.0 \times 10^{2}$ & - & - \\
\hline 02 & $1.1 \times 10^{3}$ & $4.0 \times 10^{2}$ & $2.0 \times 10^{3}$ & $1.8 \times 10^{2}$ & $2.6 \times 10^{3}$ & $1.1 \times 10^{3}$ & $5.0 \times 10^{3}$ & $1.0 \times 10^{2}$ \\
\hline 03 & $1.6 \times 10^{3}$ & $5.4 \times 10^{2}$ & $1.6 \times 10^{3}$ & $3.5 \times 10^{2}$ & $2.3 \times 10^{2}$ & $1.2 \times 10^{2}$ & $1.0 \times 10^{2}$ & $1.7 \times 10^{1}$ \\
\hline 04 & $3.2 \times 10^{4}$ & $4.0 \times 10^{3}$ & $7.2 \times 10^{2}$ & $5.2 \times 10^{2}$ & $3.0 \times 10^{2}$ & $1.0 \times 10^{2}$ & $4.0 \times 10^{2}$ & $7.0 \times 10^{1}$ \\
\hline 05 & $2.7 \times 10^{4}$ & $1.4 \times 10^{2}$ & $1.3 \times 10^{2}$ & $1.0 \times 10^{2}$ & $3.4 \times 10^{2}$ & $2.1 \times 10^{2}$ & - & - \\
\hline
\end{tabular}


used for investigating their capability to decrease the presence of microorganisms from the hands of five different individual volunteers. All of the five sanitizers had $68 \%$ alcohol as an active ingredient. In case of total viable bacteria, highest microbial load was $1.0 \times 10^{4}$ $\mathrm{cfu} / \mathrm{ml}$ and lowest was $1.8 \times 10^{3} \mathrm{cfu} / \mathrm{ml}$ of rinsed water from different volunteers before using sanitizer sample no. 01 (Table 1). After using sanitizer the loads were observed to be decreased about ten-fold. Total fungal counts were also found between $1.0 \times 10^{3}$ to $2.7 \times 10^{3}$ $\mathrm{cfu} / \mathrm{ml}$ in rinsed water before sanitation and after sanitation decreased as like bacterial count like a tenfold reduction. Pseudomonas spp. were found in lower numbers and observed to be eliminated completely in three volunteers after using sanitizer. Staphylococcus spp. were also showed ten-fold reduction in their presence after using sanitizer. For other four hand sanitizer samples, same results have been found showing at least ten fold reduction in all types of microorganisms (tables 2-5) and sometimes better results were found (Table 02: sample 02, volunteer 5; Table 04: sample 4, volunteer 4) in case of total viable bacteria reduction for only a few volunteer.

As people use instant hand sanitizers where water supply is not available, it showed some degree of decreasing capabilities but that was not enough as $68 \%$ alcohol do alone. Having $68 \%$ alcohol as active ingredient, the actual percentage might not be noted in the packaging material. Even if the concentration was same as indicated, the gel used as stabilizer and binder might be failed keep alcohol from its drying effect. As we know, instant hand sanitizer works best as an extra effort of sanitation after conventional hand washing, it cannot provide the protection we need during or before food handling and eating. As our results show that the degree of decreasing microorganisms from hand is not satisfactory to use only sanitizer during eating or in food processing area. Sanitizers work best in cleaner hands for example hands which are free from excess dirt, oil or greasy substance. It cannot be recommended to use in

TABLE 3. Microbial load in hand rinsed water of before and after using instant sanitizer sample 3

\begin{tabular}{|c|c|c|c|c|c|c|c|c|}
\hline \multirow[b]{2}{*}{ Volunteer } & \multicolumn{2}{|c|}{ Total bacterial count $(\mathrm{cfu} / \mathrm{ml})$} & \multicolumn{2}{|c|}{ Total fungal count (cfu/ml) } & \multicolumn{2}{|c|}{ Staphylococcus spp. (cfu/ml) } & \multicolumn{2}{|c|}{ Pseudomonas spp. (cfu/ml) } \\
\hline & $\begin{array}{l}\text { Hand rinsed } \\
\text { water } \\
\text { before using } \\
\text { sanitizer }\end{array}$ & $\begin{array}{c}\text { Hand rinsed } \\
\text { water after } \\
\text { using } \\
\text { sanitizer }\end{array}$ & $\begin{array}{l}\text { Hand rinsed } \\
\text { water } \\
\text { before using } \\
\text { sanitizer }\end{array}$ & $\begin{array}{c}\text { Hand rinsed } \\
\text { water after } \\
\text { using } \\
\text { sanitizer }\end{array}$ & $\begin{array}{l}\text { Hand rinsed } \\
\text { water } \\
\text { before using } \\
\text { sanitizer }\end{array}$ & $\begin{array}{c}\text { Hand rinsed } \\
\text { water after } \\
\text { using } \\
\text { sanitizer }\end{array}$ & $\begin{array}{l}\text { Hand rinsed } \\
\text { water before } \\
\text { using } \\
\text { sanitizer }\end{array}$ & $\begin{array}{c}\text { Hand rinsed } \\
\text { water after } \\
\text { using } \\
\text { sanitizer }\end{array}$ \\
\hline 01 & $4.0 \times 10^{3}$ & $1.6 \times 10^{2}$ & $1.8 \times 10^{2}$ & $1.0 \times 10^{2}$ & $1.7 \times 10^{1}$ & - & $2.0 \times 10^{1}$ & - \\
\hline 02 & $4.0 \times 10^{3}$ & $1.1 \times 10^{2}$ & $2.4 \times 10^{3}$ & $1.7 \times 10^{3}$ & $5.0 \times 10^{3}$ & $1.0 \times 10^{2}$ & $8.0 \times 10^{2}$ & $5.0 \times 10^{1}$ \\
\hline 03 & $2.4 \times 10^{4}$ & $2.6 \times 10^{3}$ & $2.3 \times 10^{2}$ & $2.2 \times 10^{2}$ & $1.0 \times 10^{2}$ & $1.7 \times 10^{1}$ & - & - \\
\hline 04 & $3.0 \times 10^{3}$ & $2.2 \times 10^{2}$ & $2.0 \times 10^{2}$ & $1.5 \times 10^{2}$ & $4.0 \times 10^{2}$ & $7.0 \times 10^{1}$ & $3.1 \times 10^{1}$ & $1.2 \times 10^{1}$ \\
\hline 05 & $2.4 \times 10^{3}$ & $1.7 \times 10^{3}$ & $3.0 \times 10^{2}$ & $2.1 \times 10^{2}$ & $1.0 \times 10^{1}$ & - & $1.7 \times 10^{1}$ & - \\
\hline
\end{tabular}

TABLE 4. Microbial load in hand rinsed water of before and after using instant sanitizer sample 4

\begin{tabular}{|c|c|c|c|c|c|c|c|c|}
\hline \multirow[b]{2}{*}{ Volunteer } & \multicolumn{2}{|c|}{ Total bacterial count $(\mathrm{cfu} / \mathrm{ml})$} & \multicolumn{2}{|c|}{ Total fungal count (cfu/ml) } & \multicolumn{2}{|c|}{ Staphylococcus spp. (cfu/ml) } & \multicolumn{2}{|c|}{ Pseudomonas spp. (cfu/ml) } \\
\hline & $\begin{array}{c}\text { Hand rinsed } \\
\text { water } \\
\text { before using } \\
\text { sanitizer }\end{array}$ & $\begin{array}{c}\text { Hand rinsed } \\
\text { water after } \\
\text { using } \\
\text { sanitizer }\end{array}$ & $\begin{array}{l}\text { Hand rinsed } \\
\text { water } \\
\text { before using } \\
\text { sanitizer }\end{array}$ & $\begin{array}{c}\text { Hand rinsed } \\
\text { water after } \\
\text { using } \\
\text { sanitizer }\end{array}$ & $\begin{array}{c}\text { Hand rinsed } \\
\text { water } \\
\text { before using } \\
\text { sanitizer }\end{array}$ & $\begin{array}{c}\text { Hand rinsed } \\
\text { water after } \\
\text { using } \\
\text { sanitizer }\end{array}$ & $\begin{array}{c}\text { Hand rinsed } \\
\text { water before } \\
\text { using } \\
\text { sanitizer }\end{array}$ & $\begin{array}{c}\text { Hand rinsed } \\
\text { water after } \\
\text { using } \\
\text { sanitizer }\end{array}$ \\
\hline 01 & $3.2 \times 10^{3}$ & $1.0 \times 10^{2}$ & $2.8 \times 10^{2}$ & $1.0 \times 10^{2}$ & $1.4 \times 10^{2}$ & $1.0 \times 10^{1}$ & $2.0 \times 10^{1}$ & - \\
\hline 02 & $1.0 \times 10^{4}$ & $2.3 \times 10^{2}$ & $2.0 \times 10^{3}$ & $1.3 \times 10^{2}$ & $2.0 \times 10^{2}$ & $1.0 \times 10^{1}$ & $8.0 \times 10^{2}$ & $5.0 \times 10^{1}$ \\
\hline 03 & $2.0 \times 10^{3}$ & $2.0 \times 10^{2}$ & $2.3 \times 10^{2}$ & $1.2 \times 10^{1}$ & $1.0 \times 10^{2}$ & $1.1 \times 10^{1}$ & $3.1 \times 10^{1}$ & $1.2 \times 10^{1}$ \\
\hline 04 & $1.5 \times 10^{3}$ & $2.7 \times 10^{2}$ & $2.5 \times 10^{2}$ & $1.2 \times 10^{2}$ & $2.0 \times 10^{3}$ & $1.0 \times 10^{2}$ & $1.7 \times 10^{1}$ & - \\
\hline 05 & $1.4 \times 10^{3}$ & $1.7 \times 10^{2}$ & $1.0 \times 10^{3}$ & $2.1 \times 10^{2}$ & $1.0 \times 10^{1}$ & - & - & - \\
\hline
\end{tabular}

TABLE 5. Microbial load in hand rinsed water of before and after using instant sanitizer sample 5

\begin{tabular}{|c|c|c|c|c|c|c|c|c|}
\hline \multirow[b]{2}{*}{ Volunteer } & \multicolumn{2}{|c|}{ Total bacterial count $(\mathrm{cfu} / \mathrm{ml})$} & \multicolumn{2}{|c|}{ Total fungal count (cfu/ml) } & \multicolumn{2}{|c|}{ Staphylococcus spp. (cfu/ml) } & \multicolumn{2}{|c|}{ Pseudomonas spp. (cfu/ml) } \\
\hline & $\begin{array}{c}\text { Hand rinsed } \\
\text { water } \\
\text { before using } \\
\text { sanitizer }\end{array}$ & $\begin{array}{c}\text { Hand rinsed } \\
\text { water after } \\
\text { using } \\
\text { sanitizer }\end{array}$ & $\begin{array}{c}\text { Hand rinsed } \\
\text { water } \\
\text { before using } \\
\text { sanitizer }\end{array}$ & $\begin{array}{c}\text { Hand rinsed } \\
\text { water after } \\
\text { using } \\
\text { sanitizer }\end{array}$ & $\begin{array}{c}\text { Hand rinsed } \\
\text { water } \\
\text { before using } \\
\text { sanitizer }\end{array}$ & $\begin{array}{c}\text { Hand rinsed } \\
\text { water after } \\
\text { using } \\
\text { sanitizer }\end{array}$ & $\begin{array}{c}\text { Hand rinsed } \\
\text { water before } \\
\text { using } \\
\text { sanitizer }\end{array}$ & $\begin{array}{c}\text { Hand rinsed } \\
\text { water after } \\
\text { using } \\
\text { sanitizer }\end{array}$ \\
\hline 01 & $2.2 \times 10^{4}$ & $1.0 \times 10^{3}$ & $2.8 \times 10^{2}$ & $2.0 \times 10^{2}$ & $1.4 \times 10^{2}$ & $1.0 \times 10^{1}$ & $2.0 \times 10^{1}$ & $1.1 \times 10^{1}$ \\
\hline 02 & $1.9 \times 10^{3}$ & $1.5 \times 10^{2}$ & $2.0 \times 10^{3}$ & $1.7 \times 10^{2}$ & $2.0 \times 10^{2}$ & $1.0 \times 10^{1}$ & $3.0 \times 10^{2}$ & $3.0 \times 10^{1}$ \\
\hline 03 & $2.0 \times 10^{3}$ & $1.7 \times 10^{2}$ & $2.3 \times 10^{2}$ & $1.4 \times 10^{1}$ & $1.0 \times 10^{2}$ & $1.1 \times 10^{1}$ & $2.5 \times 10^{1}$ & $1.2 \times 10^{1}$ \\
\hline 04 & $1.8 \times 10^{3}$ & $2.0 \times 10^{2}$ & $5.1 \times 10^{2}$ & $1.2 \times 10^{2}$ & $2.0 \times 10^{3}$ & $1.0 \times 10^{2}$ & $1.4 \times 10^{1}$ & $1.0 \times 10^{1}$ \\
\hline 05 & $1.5 \times 10^{3}$ & $1.1 \times 10^{2}$ & $1.9 \times 10^{2}$ & $1.0 \times 10^{2}$ & $1.0 \times 10^{2}$ & $1.0 \times 10^{1}$ & $2.0 \times 10^{1}$ & $1.1 \times 10^{1}$ \\
\hline
\end{tabular}


TABLE 6. Effectiveness against laboratory pathogenic isolates

\begin{tabular}{cccccc}
\hline Sanitizers & $\begin{array}{c}\text { Staphylococcus } \\
\text { aureus }\end{array}$ & $\begin{array}{c}\text { Proteus } \\
\text { vulgaris }\end{array}$ & $\begin{array}{c}\text { Escherichia } \\
\text { coli }\end{array}$ & $\begin{array}{c}\text { Pseudomonas } \\
\text { aeruginosa }\end{array}$ & $\begin{array}{c}\text { Bacillus } \\
\text { cereus }\end{array}$ \\
\hline 01 & ++ & - & + & + & - \\
02 & + & - & - & ++ & - \\
03 & + & - & - & - & - \\
04 & + & - & - & + & + \\
05 & ++ & - & + & + \\
\hline
\end{tabular}

food processing area as in that condition hands are not completely free from organic substances and greasy materials (25). Only ten-fold reduction of microbes cannot be allowed in these areas because the rest of the microbes present in hands can easily get their entrance in the food. We can use these hand sanitizers as one approach to sanitize our hands time to time basis only when there is no available water and of course our hands are not too dirty.

Instant hand sanitizers were also subjected to determine the efficacy to kill or inhibit some pathogens which are very common to cause different infections in people and can easily transmit via contaminated hands. Staphylococcus aureus, Escherichia coli, Bacillus subtilis, Pseudomonas aeruginosa and Proteus vulgaris were used in this aspect. All of the five types of sanitizers showed their capabilities to decrease the growth Staphylococcus aureus (table 6). Samole no. 01 and 05 were best among all the samples. None of these sanitizers were able to inhibit the growth of Proteus vulgaris. Sample no. 01 and 04 were able to decrease the growth of Escherichia coli. Only sanitizer sample 04 was effective against Bacillus subtilis. Sample 01, 02 and 05 were effective against Pseudomonas aeruginosa. But the degree of decreasing these pathogens were only in low range. So instant hand sanitizers can be used when the loads of microorganisms are already low after conventional hand washing with water and soap. Hand sanitizers can be an added advantage to lessen the contaminating microorganisms in day to day normal activities but not in food processing areas.

\section{CONCLUSION}

People are using instant hand sanitizers nowadays for maintenance of proper sanitation. Different types of sanitizers are used these days and alcohol based sanitizers are most popular where alcohol is the main active ingredient which kills bacteria by denaturing them. In our study it has been observed that the alcohol based sanitizers were able to decrease a low number of bacteria from hands. The same results were reflected in case of all the volunteers and all the sanitizer samples. Moreover, the pathogenic laboratory isolates also showed little positive results. So, it cannot be recommended to rely upon the instant hand sanitizers solely to give us a proper sanitary condition.

\section{REFERENCES}

1. Aiello AE, Coulborn RM, Perez V, Larson EL. 2008. Effect of hand hygiene on infectious disease risk in the community setting: a meta-analysis. Am. J. Public Health. 98: 1372-1382.

2. Tambekar DH, Shirsat SD, Suradkar SB, Rajankar PN, Banginwar YS 2007. Prevention of transmission of infectious sisease: studies on hand hygiene in health-care anmong students. Continental J. Biomed. Sci. 1: 6-10.

3. Larson EL, Hughes CA, Pyrek JD, Sparks SM, Cagataz EU, et al. 1998 Changes in bacterial flora associated with skin damage on hands of health care personnel. Am J Infect Control. 26: 513-21.

4. Winnefeld M, Richard MA, Drancourt M, Grob JJ. 2000. Skin tolerance and effectiveness of thwo hand decontamination procedures in everyday hospital use. Br. J. Dermatol. 143: 546-50.

5. World Health Organization. 2009. WHO guidelines on hand hygiene and health- care. First global patient safety challenge clean care is safer care. Section 11.2 :Plain (non-antimicrobial soap). Geneva, World Health Organization; 2009.

6. Hedberg CW, Smith SJ, Kirkland E, Radke V, Jones TF, Selman CA, et al. 2006. Systematic environmental evaluations to identify food safety differences between outbreak and non-outbreak restaurants. J. Food Prot. 69(11): 2697-2702.

7. Todd ECD, Greig JD, Barleson CA, Micheals BS. 2007. Outbreaks where food workers have been implicated in the spread of foodborne disease. Part 2. Description of outbreaks by size, severity, and setting. J. Food Prot. 70 (8): 1975-1993.

8. Todd ECD, Greig JD, Barleson CA, Micheals BS. 2007. Outbreaks where food workers have been implicated in the spread of foodborne disease. Part 3. Factors contributing to outbreaks and description of outbreaks. J. Food Prot. 70 (9): 2199-2217.

9. Larson EL. 1995. APIC guidelines for hand washing and hand antisepsis in health care settings. Amer. J. Infect. Cont. 149: 2749-2753.

10. Jones RD, Jampani HB, Jerry L, Newman JL, Lee AS. 2000. Triclosan: a review of effectiveness and safety in health care setting. Amer. J. Infect. Cont. 28: 184-196.

11. Fendler E, Groziak P. 2002. Efficacy of alcohol-based hand sanitizers against fungi and viruses. Infect. Cont. Hosp. Epidemiol. 3 (2): 61-62.

12. Fendler EJ, Ali Y, Hammond BS, Lyons MK, Kelley MB, Vowell NA. 2002. The impact of alcohol hand sanitizer use on infection rates in an extended care facility. Amer. J. Infect. Cont. 30 (4): 226-232.

13. Rotter M. 1999. Hand washing and hand disinfection. In Mayhall C.G., ed., Hospital Epidemiology and Infection Control, $2^{\text {nd }}$ ed. Lippincott Williams \& Wilkins, Philadelphia, pp.1339-1355.

14. Dyier Dl, Gernraich KB, Wadhams PS. 1998. Testing a new alcohol-free hand sanitizer to combat infection. AORN J. 68 (2): 239-241.

15. Duizer E, Bijkerk P, Rockx B, De Groot A, Twisk F, Koopmans M. 2004 Inactivation of caliciviruses. Appl. Environ. Microbiol. 70: 4538-43.

16. Hammond B, Ali Y, Fendler E, Dolan M, Donovan S. 2000. Effect of handsanitizer use on elementary school absenteeism. Amer. J. Infect. Control. 28: $340-6$.

17. Gehrke C, Steinmann J, Goroncy-Bermes P. 2004. Inactivation of Feline Calicivirus, a surrogate of Norovirus (formerly Norwalk-like viruses), by different types of alcohol in vitro and in vivo. J. Hosp. Infect. 56: 49-55.

18. Rabenau HF, Kampf G, Cinati J, Doerr HW. 2005. Efficacy of various disinfectants against SARS coronavirus. J. Hosp. Infect. 61: 107-11.

19. Sattar SA, Abehe M, Bueti AJ, Jampani H, Newman J, Hua S. 2000. Activity of an alcohol-based hand gel against human adeno-, rhino-, and rotaviruses using the finger pad method. Infect. Cont. Hosp. Epidemiol. 21: 516-519.

20. Food Standards Agency (FSA). 2014. New UK food poisoning figure published. Available at: https://www.food.gov.uk/newsupdates/news/2014/6097/foodpoisoning. Accessed on 3 February 2016.

21. National Institute of Allergies and Infectious disease (NIAID). 2014. Food borne diseases. Available at: https://www.niaid.nih.gov/topics/foodborne/ Pages/default. aspx Accessed on 3 February 2016.

22. Scallan E, Hoekstra RM, Angulo FJ, Tauxe RV, Widdowson MA, Roy SL, et al. 2011. Foodborne illness acquired in the United States - major 
pathogens. Emerg Infec. Dis. 17: 7-17

23. European Food Safety Authority (EFSA). 2011. EFSA explains foodborne disease: Food-borne zoonosis. ISBN number: 978-92-9199384-0. Fact sheet available at: http://www.efsa.europa.eu/en/topics/factsheets/factsheetfoodbornezoonose s. Assessed on 3 February 2016.

24. Food and Drug Administration (FDA). 2009. Food Code 2009 Chapter 2 - Management and Personnel - 2-301-16 Hand Antiseptics: Available at: http://www.fda.gov/Food/GuidanceRegulation/RetailFoodProtection/Food

Code/ucm181242.htm. Accessed on 3 February 2016.
25. Centers for Disease Control and Prevention (CDC). 2002. Guidelines for hand hygiene in health-care settings: recommendation of the Healthcare Infection Control Practices Advisory Committee and the HICPA/SHEA/APIC/IDSA Hand Hygiene Task Force. Morb. Mort. Week. Rep. 51: 1-56.

26. Rahman F, Noor R. 2012. Prevalence of pathogenic bacteria in common salad vegetables of Dhaka Metropolis. Bangladesh Journal of Botany. 41 (2): 159-162.

27. Hussain A, Wahab S, Zarin I, Hussain MDS. 2010. Antibacterial activity of the leaves of Coccinia indica (W. and A) of India. Adv. Biol. Res. 4 (5): 241248 . 